\title{
Resistant hypertension in times of changing definitions and treatment recommendations
}

\author{
Milad Nazarzadeh ${ }^{1,2}$, Ana-Catarina Pinho-Gomes ${ }^{1}$, Kazem Rahimi*1
}

1. George Institute for Global Health, University of Oxford, Oxford, UK

2. Collaboration Center of Meta-Analysis Research, Torbat Heydariyeh University of Medical Sciences, Torbat Heydariyeh, Iran.

*Corresponding author: Kazem Rahimi, George Institute for Global Health, University of Oxford, Oxford, UK; kazem.rahimi@georgeinstitute.ox.ac.uk

Hypertension is one of the most prevalent conditions in the world. Despite evidence for the beneficial effects of blood pressure (BP) lowering across a range of patient groups [1], treatment and control rates remain suboptimal in high and low income countries alike.[2] To alert physicians to the fact that some patients with hypertension might benefit from specialist assessment for possible underlying causes or more advanced treatment recommendations, clinical practice guidelines introduced a new sub-category of hypertension called resistant hypertension (Figure). The ESC guidelines defined resistant hypertension as the failure to achieve BP 'control' to levels below 140/90 mmHg despite treatment with 3 antihypertensive medications with complementary mechanisms of action (with at least one diuretic).[3] The AHA guidelines adopted an extended definition to also include patients with 4 or more antihypertensive medications, even when BP was controlled.[4] To acknowledge the fact that 'apparent' resistant hypertension might turn out to be spurious (because of measurement error, white coat effect or poor patient adherence with treatment), a further sub-categorisation into 'true' resistant and 'pseudo' resistant was made in clinical studies (Figure). 
In the current issue of Heart, Noubiap et al.[5] report the findings of a meta-analysis that estimates the prevalence of apparent resistant, true resistant and pseudo-resistant hypertension among treated hypertensive patients. By including 91 cohort or cross-sectional studies with over 3.2 million hypertensive patients in total (most of them from high-income countries), they estimated the prevalence of resistant hypertension across different geographic regions. Overall, the unadjusted prevalences of apparent resistant, true resistant and pseudo resistant in the entire population of patients with treated hypertension were $14.7 \%, 10.3 \%$, and $10.3 \%$, respectively. However, more important than the absolute estimates reported, the significant and largely unexplained statistical variability in this meta-analysis highlights the challenges of measurement and interpretation of the burden of resistant hypertension.

One of the main challenges is the definition of resistant hypertension itself. As the systematic review by Noubiaq et al. reveals, the definitions used to classify patients with apparent resistant hypertension varied substantially between studies, with some adopting the AHA and others the ESC definition. Some of the studies were based on selected patient populations and might not have been representative of the treated or total hypertensive population. Thus, it is perhaps not very surprising that estimates for resistant hypertension ranged from $5 \%$ to $35 \%$ in the identified studies. Relatedly, the overall pooled estimate of $14.7 \%$ is likely to be even less reliable when put in the context of the recently changed AHA guidelines which have lowered the threshold for resistant hypertension to $130 / 80 \mathrm{mmHg}$.[6] This together with recent evidence from clinical trials suggesting that a strategy of combining multiple classes of anti-hypertensives at low doses might yield better BP reductions than the conventional approach of maximum up-titration of fewer agents, [7] the concept of labelling patients to have resistant hypertension simply because they are prescribed 3 or 4 antihypertensives seem to have become obsolete. 
As Noubiap et al. point out, it is more important to know what the true prevalence of resistant hypertension after pseudo-resistance has been excluded than to have an overall estimate of apparent resistant hypertension. However, only 12 studies involving about 75,000 individuals were identified that had reported the prevalences of true and pseudo resistant hypertension and these were mainly were hospital-based and relied on office-based BP measurements. Not only was the range of estimates among studies very wide, but the proportion of patients having pseudo vs true resistant hypertension was very different among the studies included. For instance, in some studies $2 / 3$ of the patients had pseudo resistant hypertension and in others the proportions were reversed, with over $2 / 3$ having true resistant hypertension (see Figure 2 of the paper). Accurate determination in clinical practice and research studies of whether resistant hypertension is actually attributable to "a refractory disease or a refractory patient" [8] is complex and not always possible. Surveys such as that conducted by Tomaszewski et al. using liquid chromatography-tandem mass spectrometry (HP LC-MS/MS) urine analysis showed that at least a quarter of patients did not comply with BP-lowering treatments.[9] Thus, the prevalence of true treatment-resistance is also likely to change as more sophisticated methods for exclusion of pseudo-resistance are adopted.

In conclusion, Noubiap et al. remind us that resistant hypertension affects a sizable proportion of patients with treated hypertension and about half of them have true resistant hypertension that requires more aggressive BP management to avoid the deleterious consequences of persistently elevated BP. The study also reveals that the concept of resistant hypertension, particularly 'controlled' resistant hypertension and numbers of drugs used, may deserve to be revised in keeping with the recent decrease in diagnostic thresholds and multidrug low-dose treatment recommendations for hypertension. 
References

1 Ettehad D, Emdin CA, Kiran A, et al. Blood pressure lowering for prevention of cardiovascular disease and death: A systematic review and meta-analysis. Lancet 2016;387. doi:10.1016/S0140-6736(15)01225-8

2 Rahimi K, Emdin CA, MacMahon S. The epidemiology of blood pressure and its worldwide management. Circ Res 2015;116:925-36. doi:10.1161/CIRCRESAHA.116.304723

3 Mancia G, Fagard R, Narkiewicz K, et al. 2013 ESH/ESC guidelines for the management of arterial hypertension: the Task Force for the Management of Arterial Hypertension of the European Society of Hypertension (ESH) and of the European Society of Cardiology (ESC). Eur Heart J 2013;34:2159-219. doi:10.1093/eurheartj/eht151

4 Calhoun DA, Jones D, Textor S, et al. Resistant Hypertension: Diagnosis, Evaluation, and Treatment: A Scientific Statement From the American Heart Association Professional Education Committee of the Council for High Blood Pressure Research. Hypertension 2008;51:1403-19. doi:10.1161/HYPERTENSIONAHA.108.189141

5 Jean Jacques Noubiap, Jobert Richie Nansseu UFN, Paule Sandra Sime IF, Joel BJ. Global prevalence of resistant hypertension: a meta-analysis of data from 3.2 million patients. Heart 2018.

6 Whelton PK, Carey RM, Aronow WS, et al. 2017 ACC/AHA/AAPA/ABC/ACPM/AGS/APhA/ASH/ASPC/NMA/PCNA Guideline for the Prevention, Detection, Evaluation, and Management of High Blood Pressure in Adults. J Am Coll Cardiol 2017;:24430. doi:10.1016/j.jacc.2017.11.006

7 Chow CK, Thakkar J, Bennett A, et al. Quarter-dose quadruple combination therapy for initial treatment of hypertension: placebo-controlled, crossover, randomised trial and systematic review. Lancet (London, England) 2017;389:1035-42. doi:10.1016/S0140-6736(17)30260-X

8 Sternlicht H, Bakris GL. Resistant Hypertension: A Refractory Disease or Refractory Patient. Hypertens. (Dallas, Tex. 1979). 2017;69:582-3. doi:10.1161/HYPERTENSIONAHA.117.08924

9 Tomaszewski M, White C, Patel P, et al. High rates of non-adherence to antihypertensive treatment revealed by high-performance liquid chromatographytandem mass spectrometry (HP LC-MS/MS) urine analysis. Heart 2014;100:855-61. doi:10.1136/heartjnl-2013-305063 
